\title{
Surgery in epilepsy associated with focal lesions in childhood
}

Marie Bourgeois, M.D., Christian Sainte-Rose, M.D., Arielle Lellouch-Tubiana, M.D., Conor Malucci, M.D., F.R.C.S., Francis Brunelle, M.D., Wirginia Maixner, F.R.A.C.S., Giuseppe Cinalli, M.D., Alain Pierre-Kahn, M.D., Dominique Renier, M.D., Michel Zerah, M.D., Jean-François Hirsch, M.D., Francoise Goutières, M.D., and Jean Aicardi, M.D.

Unit of Pediatric Neurology, and Department of Pediatric Neurosurgery, Pathology, and Pediatric Radiology, Hôpital Necker-Enfants Malades, Paris, France

Surgery in children with epilepsy is a new, evolving field. The important practical issues have been to define strategies for choosing the most suitable candidates, as well as the type and the optimum timing of epilepsy surgery. This study was undertaken to illustrate these points.

To identify the factors that correlated with outcome, the authors analyzed a series of 200 children (aged 1-15 years, mean 8.7 years) who underwent surgery at the Hôpital Necker-Enfants Malades between 1981 and 1996. In 171 cases (85.5\%) the epilepsy was medically refractory and was associated with focal cortical lesions. Surgery consisted of resection of the lesion without specifically attempting to identify and remove the "epileptogenic area."

In the group of children whose seizures were medically refractory, the mean follow-up period was 5.8 years. According to Engel's classification, $71.3 \%$ of these children became seizure free (Ia), whereas $82 \%$ were in Class I. A multivariate statistical analysis revealed that among all the factors studied, the success of surgery in a patient in whom there was a good clinical-electroencephalogram-imaging correlation, depended on the patients' having undergone a minimally traumatic operation, a complete resection of the lesion, and a short preoperative seizure duration.

After the surgical control of epilepsy, behavior disorders were more improved (31\% of all patients) than cognitive function $(25 \%)$. The patient age at onset, duration and frequency of seizures, intractability of the disease to therapy, and seizure characteristics, were correlated with cognitive, behavioral, and academic performance pre- and postoperatively. Multivariate statistical analysis revealed that cognitive dysfunction correlated highly with the duration of epilepsy prior to surgery, whereas behavioral disorders correlated more with seizure frequency.

These data must be taken into account when selecting patients for surgical treatment and when deciding the timing of surgery. Early surgical intervention allows for optimum brain development.

Key Words * epilepsy surgery* lesionectomy * children * lesional epilepsy * focal lesion* outcome 
In the last decade, epilepsy surgery has evolved into an effective alternative treatment for intractable partial epilepsy in children.[1,5,11,26,31,61] Important considerations that have contributed to this trend were the appreciation of the adverse effects of seizures on the developing brain, the plasticity of the young brain in response to surgery, and the hope of some developmental "catch up" after successful early surgical treatment. $[8,9,54]$ However, the natural history of epilepsy in childhood is not yet very well known. Thus, selection criteria for epilepsy surgery remain incompletely defined, partly because of an existing historical belief that children may grow out of their epilepsy. It is now recognized that epilepsy related to an underlying structural lesion is poorly responsive to medication, and is unlikely to be cured spontaneously.[2,12,31,37,39,42,44,55,68,72,80,82] Recent advances in neuroradiology allow more frequent identification and better definition of a lesion in children who suffer from partial seizures.[17] Low-grade tumors, dysplastic lesions, focal neuronal migration disorders, vascular malformations, and secondary scar lesions are all important etiological factors in children with partial seizure disorders. Whether the severity and the type of the seizures are predictive of the cause of the epileptogenic lesion is still under debate, as well as whether a separate study of the epileptogenic activity caused by these pathological entities would be appropriate.

The type of preoperative investigations and the choice and timing of surgery also remain open to discussion.[10,11] For instance, issues such as secondary onset of an epileptic seizure and the potential beneficial effect of early surgical intervention have attracted increasing attention recently.

Over the last 16 years at Hôpital Necker Enfants-Malades, a series of children who presented with epilepsy were found to harbor structural lesions and were considered for surgical treatment. We report a series of 200 children who underwent "lesionectomy." Despite the inherent limitations of a retrospective analysis, the results of our study illustrate a number of points on the management of epilepsy related to structural lesions.

\section{CLINICAL MATERIAL AND METHODS}

\section{Patient Population}

Over a 16-year period, 200 children with partial seizures and cortical lesions were treated surgically at Hôpital Necker Enfants-Malades in Paris. At presentation, in 171 of these patients epilepsy was refractory to medical therapy, whereas in 29 patients seizures were controlled by treatment. To maintain clarity of the issues, these two groups will subsequently be analyzed separately.

Patients with malignant neoplasm, cyst, arteriovenous malformation, isolated temporal sclerosis, microdysgenesis, Rasmussen encephalitis, and neuronal heterotopia were not included. Patients with hemimegalencephaly or other diffuse abnormalities were also excluded. This selection process was conducted to avoid the possible effect that any associated malformation might have on the outcome.

There were 114 boys and 86 girls whose mean age at presentation was 8.7 years (range 10 months-15 years). All patients underwent scalp electroencephalography (EEG), neuroimaging, and psychometric evaluation. Epileptogenic lesions were identified by the presence of multiple features, including semiology of seizures suggesting localized onset, focal interictal or ictal EEG findings, and a well-identified lesion on computerized tomography and/or magnetic resonance (MR) imaging. All patients underwent "lesionectomy," that is, the surgical excision of the lesion that was demonstrated on radiological examination. Regular follow-up evaluation was performed at 6-month intervals for the first 2 
years after surgery and at 1-year intervals thereafter. Only children who underwent a minimum follow-up duration of 18 months were included in this study.

\section{Clinical Assessment}

Patient age at the onset of seizures and at operation, duration of seizures, type and frequency of seizures, neurological findings, location of the lesion, and surgical outcome were recorded in each patient. Cognitive and psychological statuses and their relation to academic performance were evaluated at presurgery and at follow up. Details of problems during pregnancy and delivery, childhood febrile convulsions, and a history of status epilepticus were also noted. The presenting features of seizures and their important chronology were carefully studied because they were important in establishing the location of the epileptic focus in children being considered for epilepsy surgery.

Seizure frequency was divided into four groups: daily, weekly, monthly, or less than monthly if patients experienced an average of at least one seizure per day, week, month, or less frequently, respectively.

Intelligence quotients (IQs) determined by the Wechsler test were divided into "normal" (either verbal or performance IQ over 90), "slightly retarded" (scores between 90 and 70), "moderately retarded" (scores between 70 and 50), or "severely retarded" (scores under 50). Those children with a normal IQ were subdivided according to the presence or absence of learning disabilities such as memory deficit, limited attention span, poor speed factor, and problem-solving ability.

Data on behavior and psychosocial development were divided into four groups: normal behavior, minor psychological impairment (inhibition, anxiety), hyperactivity, and psychosis. School performance was also studied, and children were divided into the following groups: younger than six years of age (preschool age), normal schooling, moderate difficulties with a school delay of less than 2 years, severe difficulties with a delay of over 2 years, and no possibility of schooling.

Surgical outcome was evaluated to obtain data on seizure control after at least 18 months of follow up and was graded according to the classification of Engel.[77] Surgical outcome was also evaluated according to neurological, cognitive, and psychosocial outcome.

\section{Electroencephalography Studies}

The results of interictal scalp EEG monitoring was assessed for background activity, slow-waves, focal spikes, and generalized paroxysmal discharges in each child. Background activity was classified as normal, asymmetrical, and slow; focal spikes as localized, regional, multifocal, or hemispheric with or without focal, regional, or hemispheric slow waves; and generalized paroxysmal discharges as diffuse irregular spike-and-wave activity or multiple spike-and-wave activity but excluding diffuse high-voltage slow bursts. Repeated EEG was performed in all patients. Neither sphenoidal EEG nor invasive EEG recordings were performed.

\section{Imaging Studies}

Computerized tomography scans with and without addition of contrast material were obtained in all patients and gadolinium-enhanced MR images in 152 patients. Postoperative magnetic resonance imaging including $\mathrm{T}_{2}$ - weighted imaging was performed in all patients. We defined "surgical injury," to be a significant area of brain atrophy adjacent to the resection margin that was very likely to be related to excessive retraction and/or vascular damage. Neither single-photon emission computerized tomography 
(SPECT) nor position-emission tomography (PET) scanning was performed.

\section{Surgical Procedure}

Lesionectomy was performed in all children, and included resection of the lesion and some of the surrounding cortex, depending on the location and the type of the lesion. Excision of a cortical lesion developing in a gyrus only involved removal of the lesion itself when it was situated within eloquent cortex, and included the adjacent cortex up to the sulcal boundaries when within noneloquent areas. However, although the resected area was, in most cases, larger than the lesion itself, no specific attempt was made to remove the epileptogenic area that was identified on preoperative invasive ictal recordings. In temporal lobe lesions, the resection proceedure did not include a formal temporal lobectomy except in those patients in whom the lesion largely involved the whole temporal lobe. All operations were performed after induction of general anesthesia, and intraoperative electrocorticography was not performed. Intraoperative localization methods included ultrasonography, stereotaxy, and, more recently, image-guided computer-assisted surgery.[3,30]

\section{Histopathological Studies}

All resected specimens were examined both macroscopically and histologically, and appropriate staining was performed when tumor was found. Particular attention was paid to specimens previously diagnosed as low-grade gliomas, which were reclassified as dysembryoplastic neuroectodermal tumors (DNETs) when appropriate, according to recently revised criteria.[25,75]

\section{RESULTS}

\section{Patients With Medically Controlled Seizures}

Medical treatment was attempted in all patients prior to surgery. At the time of operation, seizure control had been achieved in 29 children (14\% of the cases) for several weeks or months. Surgery was indicated in this group if the neuroradiological appearance of the lesion suggested a tumor (eight cases) or a vascular lesion (21 cases with cavernomas).

The majority of the patients in this group experienced epilepsy at a late age of onset (13 patients after 9 years of age) and of a short duration of seizures ( $<6$ months in 19 cases). These children had rare seizures, with only two or three occurring during their short history of epilepsy.

All of these patients, except for two, were cured by excision of their lesion. Of the two patients who still experience seizures, one suffered a middle cerebral artery thrombosis and the other who harbored a benign glial lesion adjacent to the central gyrus underwent an incomplete resection. This latter patient has undergone reoperation, but the follow-up period has been too short to include results.

\section{Preoperative Status in Patients With Medically Refractory Epilepsy}

In 171 children, epilepsy was not controlled by a trial of single or combination first-line conventional antiepileptic drugs and proved intractable to medical treatment. These patients constitute the core population of this study.

Clinical Features. No pre- or perinatal predisposing factors for seizures were identified in any of the patients. Three patients had suffered viral encephalitis with no apparent consequences. Of those with medically refractory epilepsy, 18 children $(11 \%)$ had a history of febrile convulsions. 
Age at Onset of Seizures. Seizures began between the ages of 6 weeks and 14 years. It is of interest that $43 \%$ of the children were younger than 3 years of age at the time of seizure onset. The mean age at operation was 8.3 years (range 10 months-15 years). There was, therefore, an average time interval of 2.5 years (1 month-12 years) between the onset of seizures and surgery.

Duration of Epilepsy. Forty-two percent of the children had experienced seizures for greater than 2 years to a maximum of 15 years. One-fifth of the children had experienced seizures for more than 6 years, which reflects the early difficulty in diagnosis and imaging. Patients in whom diagnosis was made within the last 5 years tended to have a shorter duration of epilepsy, which reflects an increasing tendency to use MR imaging.

Seizure Frequency. Daily seizures were noted in $40 \%$ of patients, and there was a tendency for seizures to appear in clusters of 10 to 20 per day and to last up to a few days. Weekly and monthly seizures were noted in $27 \%$ and $32 \%$ of the cases, respectively.

Seizure Type. Seizures were divided into the following groups: simple partial seizures (23.4\% of cases), complex partial seizures (18.7\% of cases), simple partial with secondary generalized tonic-clonic seizures $(9.4 \%$ of cases), complex partial with secondary generalized tonic-clonic seizures (12.9\% of cases), mixed simple and complex partial seizures such as versive or unilateral motor seizures, in addition to complex partial seizures ( $28.1 \%$ of cases), "generalized seizures" ( $4 \%$ of cases), and infantile spasms $(3.5 \%$ of cases).

Presenting Features of Seizures. Focal sensorimotor activity was documented in 70 children, and 53 patients experienced seizures that began with a subjective sensation. In three patients seizure consisted only of a subjective sensation. In 72 patients "psychomotor arrest" was the presenting feature; this was sometimes associated with inappropriate behavior, preceded by a subjective sensation in 33 patients and by somatosensory manifestations in 39 patients. Secondary falls were preceded by a subjective sensation with impairment of consciousness in 13 patients, somatosensory manifestations in four patients, and subjective sensation and somatosensory manifestations with impairment of consciousness in 35 patients. Six children suffered infantile spasms during the 1st year of life, but subsequently their pattern evolved predominantly into asymmetrical motor phenomena with complex partial seizures.

Location of the Lesion. Figure 1 shows the location of the lesions. There was an equal side distribution. 


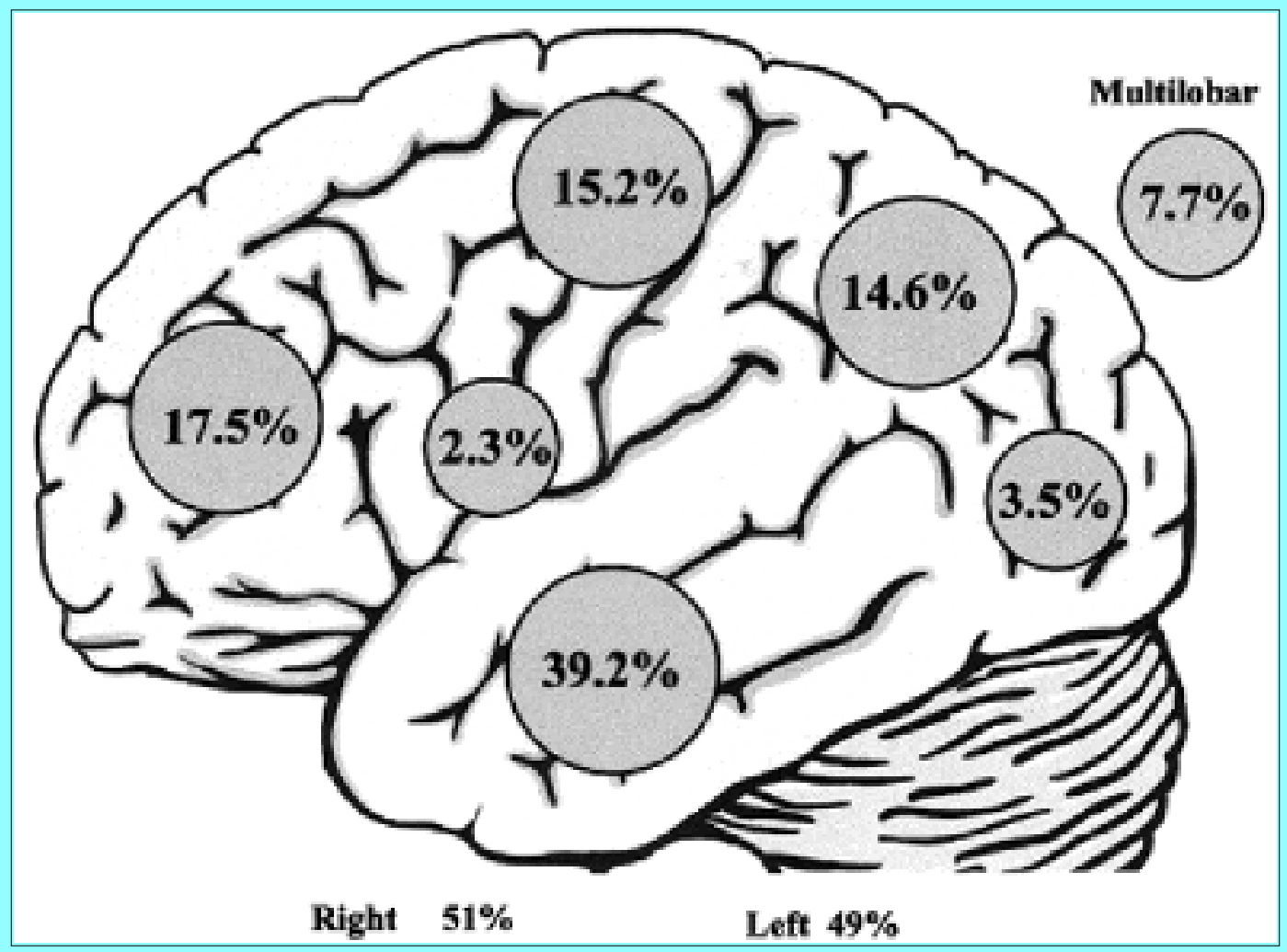

Fig. 1. Drawing illustrating the location of the lesion as visualized on neuroimaging studies.

Electroencephalography. Interictal abnormalities are shown in Fig. 2. Epileptiform abnormalities were focal and strictly concordant with radiologically demonstrated lesions in $80 \%$ of the cases. Ictal activity, recorded in $45 \%$ of the children, demonstrated a good correlation between the location of the EEG abnormalities and the location of the lesion. There was no significant correlation between EEG abnormalities and the cause of the lesion.

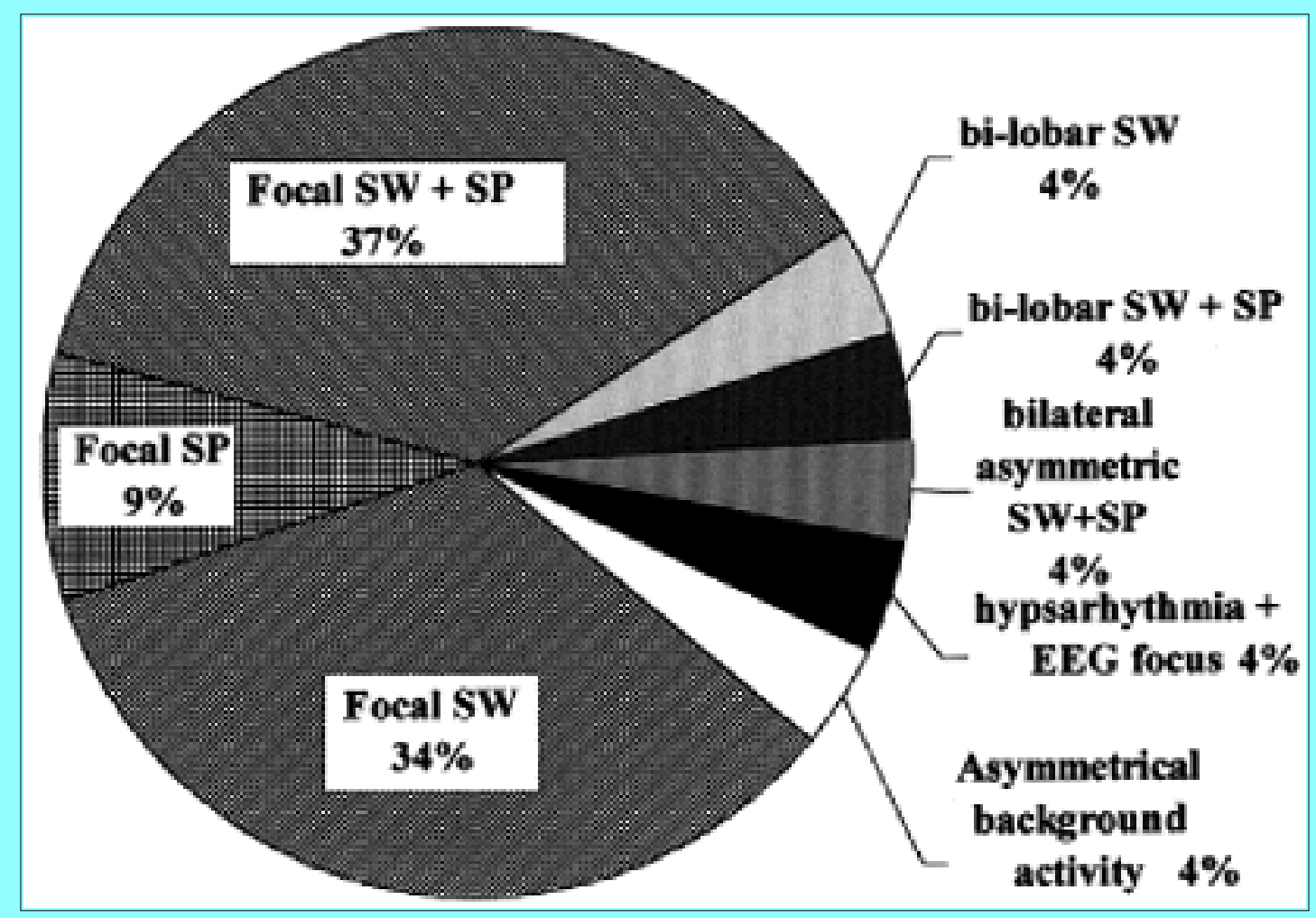

Fig. 2. Pie chart showing the interictal abnormalities, which were focalized in $80 \%$ of the 
cases, and either spikes (SP) or slow-wave activity (SW), or a combination of both.

Imaging Abnormalities. Neuroradiological findings were heterogeneous in this series. Although the appearance of a cavernous angioma or of a localized Sturge-Weber syndrome was often typical, neuroimaging did not accurately discriminate brain tumors from dysembryoplastic lesions.

Calcifications, cysts, and contrast enhancement were not found to be specific to any of these diseases. Moreover, as similarly reported by others,[52] an association between an area of focal cortical dysplasia and a dysembryoplastic lesion or a ganglioglioma was observed in several of our cases.

Neurological Examination. Preoperative neurological status in all children was normal, except in five who experienced mild motor deficits and in four others with visual field defects (hemianopsia or quadrantanopsia).

Preoperative Cognitive Status. All patients were evaluated prior to surgery. Developmental milestones were normal in $52 \%$ of the children. In 30 children learning disabilities were demonstrated.

Serial psychometric testing results were available in all cases and showed evidence of progressive cognitive decline in 30 children, particularly in verbal skills. School performance was recorded for 125 children who were over the age of 6 years. A less-than-normal status was observed in one-third of the cases.

Preoperative neuropsychological and cognitive status was assessed in relation to the patient's age at onset of seizures, duration of seizures, frequency of seizures, mode of presentation, location of lesion, and identification of those factors that could have influenced the preoperative status in these children. Both preoperative cognitive status and psychological status were shown to have a significant partial correlation ( $\mathrm{p}<0.05$ ) with age at seizure onset, duration of epilepsy preoperatively, seizure frequency, loss of consciousness during seizures, as well as the location and the type of lesion. However, a logistical regression (forward stepwise) analysis conducted to examine all these factors revealed that preoperative cognitive status was mainly dependent on the duration of seizure activity before surgery (Fig. 3 upper) whereas psychological status was mainly influenced by seizure frequency (Fig. 3 lower). In children with a long history of seizures refractory to anticonvulsant medication, a marked decline in cognitive function was demonstrated, whereas children with frequent attacks presented with behavioral disorders and psychosocial disability. 


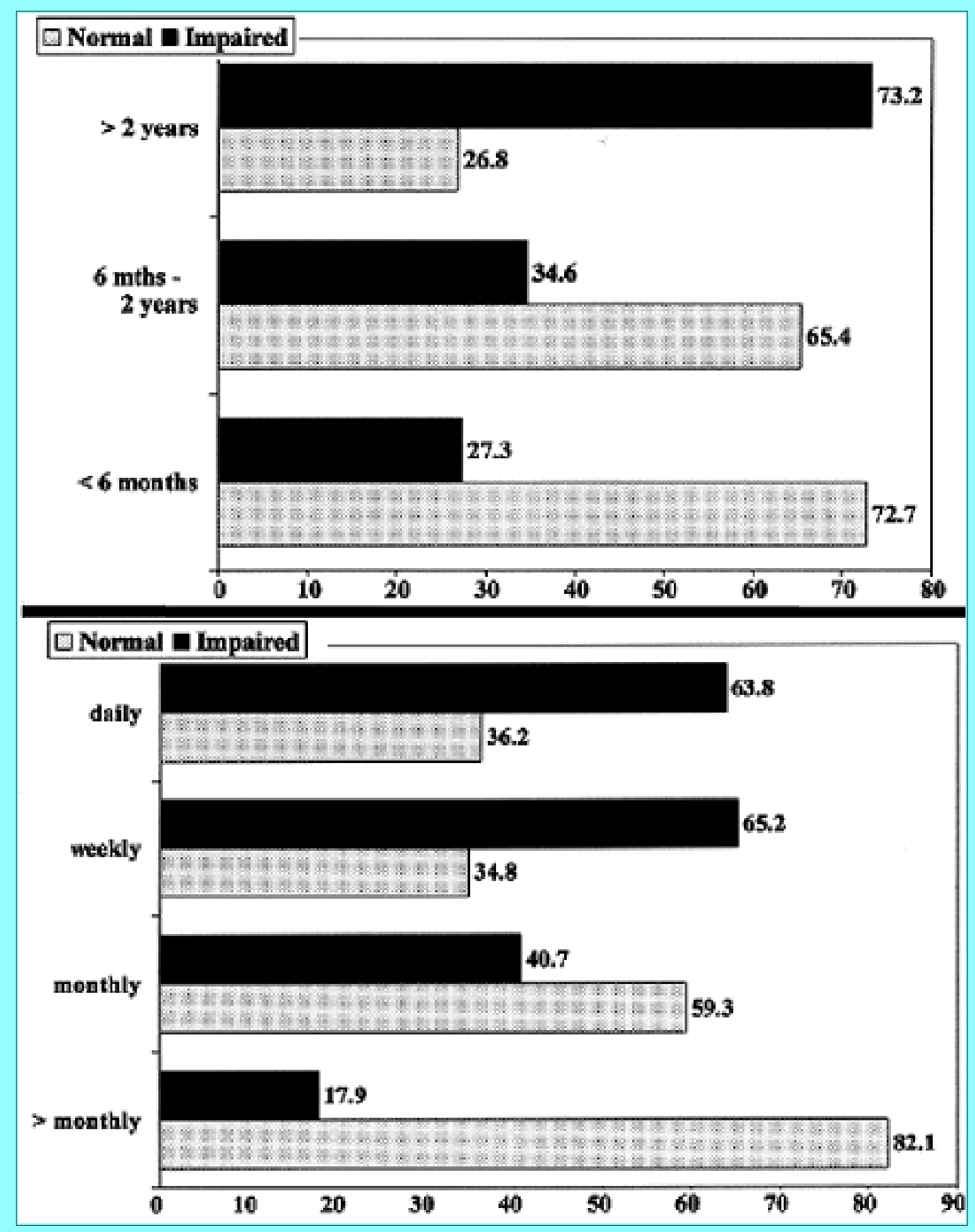

Fig. 3. Bar graphs. Upper: The preoperative cognitive status in children was strongly correlated with the duration of epilepsy. Two-thirds of the patients who experienced seizures for less than 6 months had a normal cognitive status, whereas two-thirds of the patients who experienced seizures for more than 2 years were intellectually impaired. Lower: Psychological status was mainly influenced by seizure frequency. The more frequent the seizures, the higher the incidence of behavioral disorders. Numbers within the graph are expressed as percentages.

Types of Lesions. In Table 1 the different types of lesions, which were classified after histopathological examination of the resected specimens, are outlined. Of some interest are the DNETs. In the 32 patients in whom DNETs were identified, eight DNETs had been previously diagnosed as low-grade gliomas and 
were subsequently reclassified.

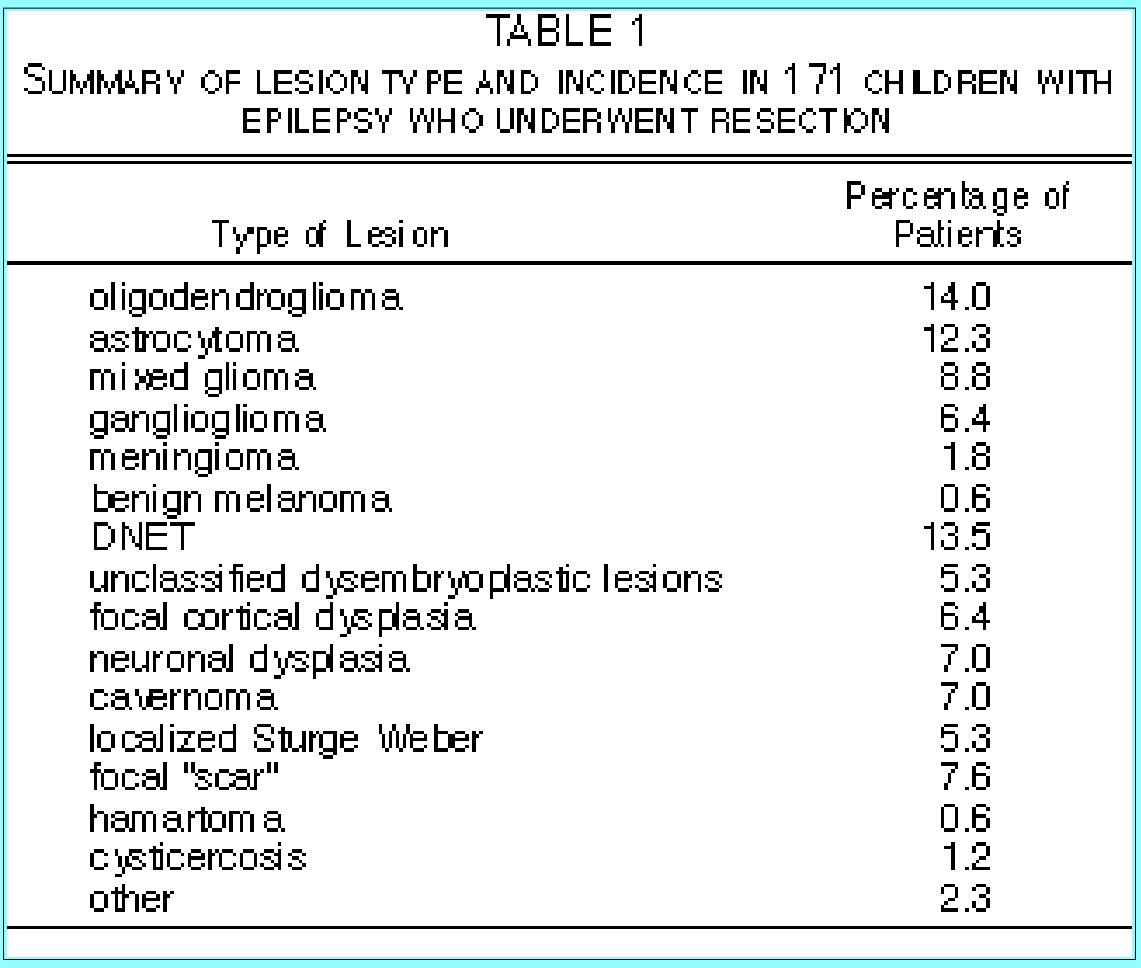

\section{Postoperative Status}

First Surgical Procedure. A complete lesionectomy was achieved in 113 (66.1\%) of 171 cases (Fig. 4) and a partial resection in $58(33.9 \%)$ of 171 cases.

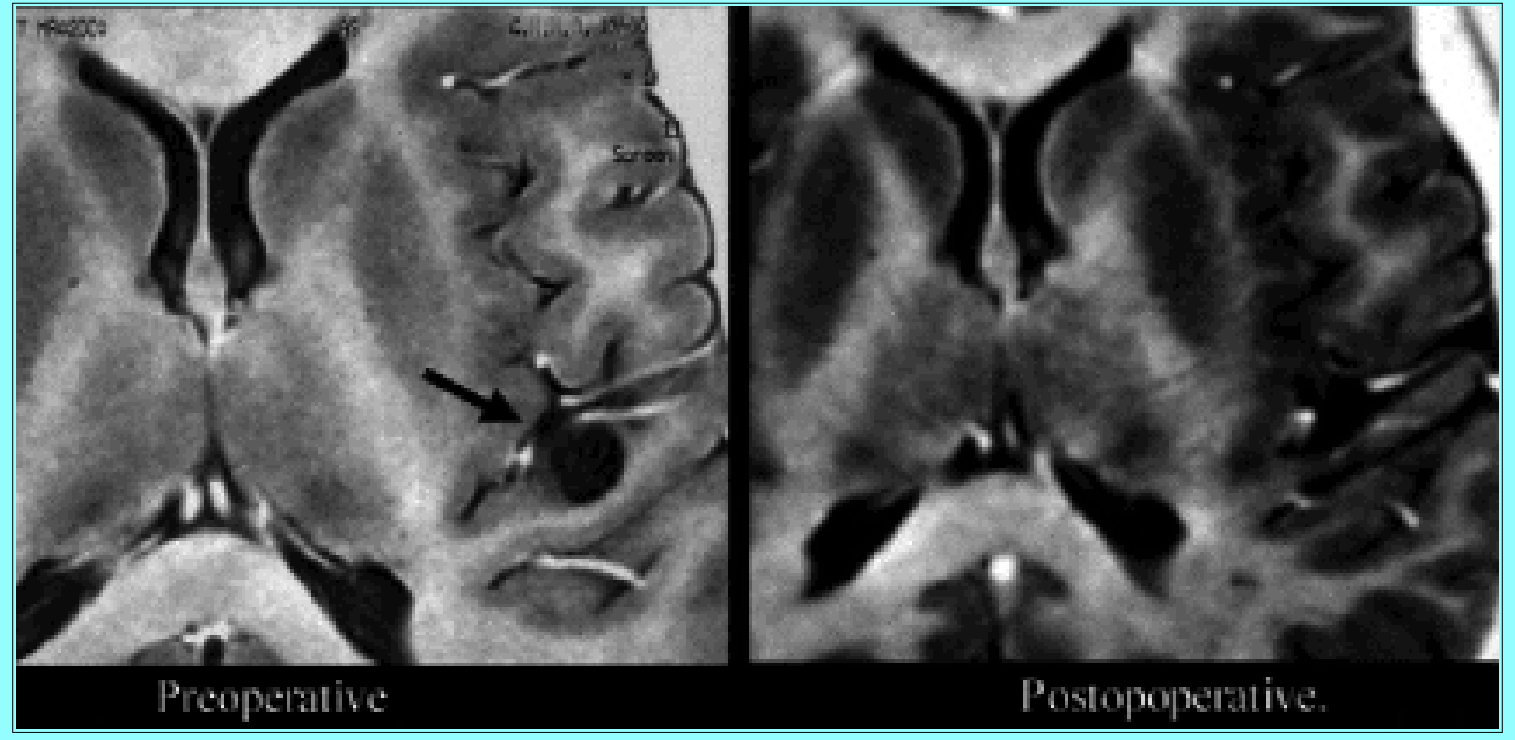

Fig. 4. Pre- and postoperative $M R$ images ( $\mathrm{T}_{1}$-weighted and contrast-enhanced) demonstrating a lesionectomy in a child who harbored a left precentral astrocytoma (arrow).

Immediate operative deficits included speech, visual, or motor deficits in 41 (24\%) of 171 children. Mild motor deficits caused by tissue retraction close to functional areas were transient in 21 children in the immediate postoperative period, whereas five patients developed a permanent deficit after sustaining either a surgery-related injury to the surrounding brain or after resection of a structural lesion from the motor cortex. Of the seven patients who developed a new postoperative quadrantanopsia, which was 
related to the removal of a lesion located in the temporal or occipital lobe, two children recovered. Three children developed nominal aphasia that gradually improved to complete recovery within a few weeks. No postoperative infections, intracranial hemorrhages, or deaths occurred. Seizure recurrence was observed in 63 children (39\%) with a mean delay of 15 months (range1 day-10 years).

Reoperation. A second operation was performed in 30 children, following a mean interval of 2.5 years. Reoperation was indicated in four patients in whom tumors recurred with renewed onset of seizure activity and in 26 patients with a residual lesion in whom the original surgery failed to improve seizure frequency. This second lesionectomy was partial in 11 and complete in 19 patients.

Of these 30 patients, deficits related to second surgery included a transient motor deficit in four cases because the resection extended close to the motor cortex. Permanent quadrantanopsia was observed in three cases.

Recurrence of seizures was noted after the second operation in 12 of these children, with a mean delay of 8 months (range 5 days- 4 years).

Outcome. The mean postoperative follow-up period was 5.2 years (range 18 months-17 years), with 47 (27.5\%) of 171 children undergoing follow up for more than 6 years.

Postoperative neuroradiological imaging demonstrated an area of focal atrophy clearly related to surgical injury or vascular damage in 16 children. Evidence of a residual lesion was visible in 41 cases.

Seizure Outcome. The overall outcome is shown in Table 2, with the status in $71.3 \%$ of the children classified as Engel's Grade Ia. The actuarial probability to be seizure free after surgery (Grade Ia) was $85.5 \%$ at 1 year and $61.2 \%$ at 10 years (Fig. 5).

\begin{tabular}{|cc|}
\hline \multicolumn{3}{|c|}{ TABLE 2 } \\
ENGEL CLASSFCATION AT A MEAN FOLLOW UP \\
OF 5.2 VEARS
\end{tabular}




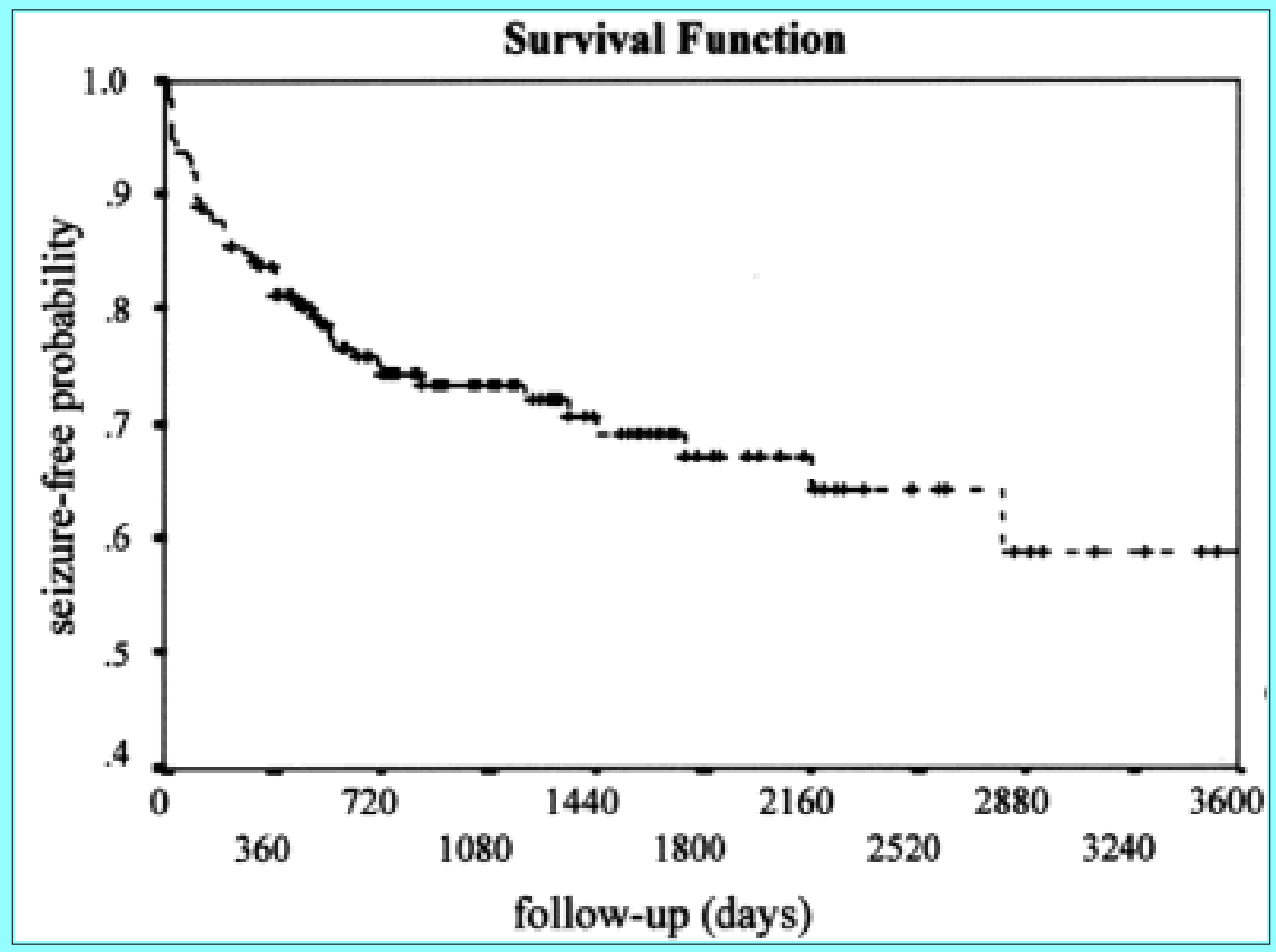

Fig. 5. Graph showing actuarial probability of becoming seizure free after removal of the lesion in 171 children with medically refractory epilepsy.

With regard to epilepsy, only nine patients $(5.2 \%)$ received no benefit from surgery. To date, medical treatment has been discontinued in $75(43.9 \%)$ of 171 children after a mean interval of 18 months. Of the remainder, 30 children (17.5\%) have been receiving a reduced course of antiepileptic medication.

Of the six children in whom a diagnosis of infantile spasms was made, four were seizure free (two without medication), and in one patient the seizure frequency had significantly decreased from the preoperative state.

Electroencephalography. Results of postoperative sleep and awake EEG monitoring were normal in 87 children $(50.9 \%)$. The mean time for normalization of traces was 12 months. Postoperative scalp EEG monitoring indicated abnormal recordings in 84 patients: unilateral spikes over the operative site in five, focalized slow-wave activity in 49 , unilateral spikes or spike and slow wave activity in 22 , and more diffuse bilateral abnormalities in the remaining cases. Children in whom the postoperative EEG demonstrated abnormal finding had a significantly worse seizure outcome than those with normal recordings (Table 3 ). 


\begin{tabular}{|c|c|c|}
\hline $\begin{array}{r}\text { CORRELATION OF POS } \\
\text { CEREBRAL INURV, AND } \\
\text { SEIZU }\end{array}$ & $\begin{array}{l}3 \\
\text { TIVE EEC } \\
\text { AGE OF R } \\
\text { OME* }\end{array}$ & $\begin{array}{l}\text { RGCAL } \\
\text { TION WITH }\end{array}$ \\
\hline \multirow[b]{2}{*}{ 'Variatle } & \multicolumn{2}{|c|}{ No. of Patients (\%) } \\
\hline & Enged la & Seizures \\
\hline \multicolumn{3}{|l|}{$\mathrm{EEG}$} \\
\hline normal & 94.3 & 5.7 \\
\hline abnormal & 46.4 & 53.6 \\
\hline \multicolumn{3}{|l|}{ surgical ceretral injury } \\
\hline $\begin{array}{l}\text { none } \\
\text { injury }\end{array}$ & $\begin{array}{r}77.4 \\
6.3\end{array}$ & $\begin{array}{l}22.6 \\
938\end{array}$ \\
\hline \multicolumn{3}{|l|}{ degree of resection } \\
\hline complete & 85.4 & 14.6 \\
\hline incomplete & 24.4 & 75.6 \\
\hline
\end{tabular}

Multivariate Analysis. A multivariate analysis (forward logistical regression) was performed to identify those factors that potentially correlate with persistent seizures. Factors included in the model were patient age at onset of seizures, characteristics of seizures, seizure frequency, location of lesion, duration of seizures, degree of resection, and surgical damage. Surgical cerebral damage was the first factor determined in the model. All 16 children but one, who sustained a surgically induced cerebral injury, continued to experience seizures after surgery (Table 3). The second factor was the degree of resection. Seizure control following partial resection of the lesion was observed in only $24.4 \%$ of the patients, whereas $95.6 \%$ of those children who underwent a complete tumor resection became seizure-free. An incomplete resection occurred for two main reasons: penetration into eloquent cortex such as speech or motor areas and poor macroscopic differentiation of the margins of the lesion (Table 3).

Children with a long preoperative duration of seizures were more likely to suffer from persistent seizures. Indeed, the mean duration of seizures was significantly longer among children with persistent seizures after surgery as compared with those who were seizure free (1562 days and 950 days, respectively) (Fig. $6)$. 


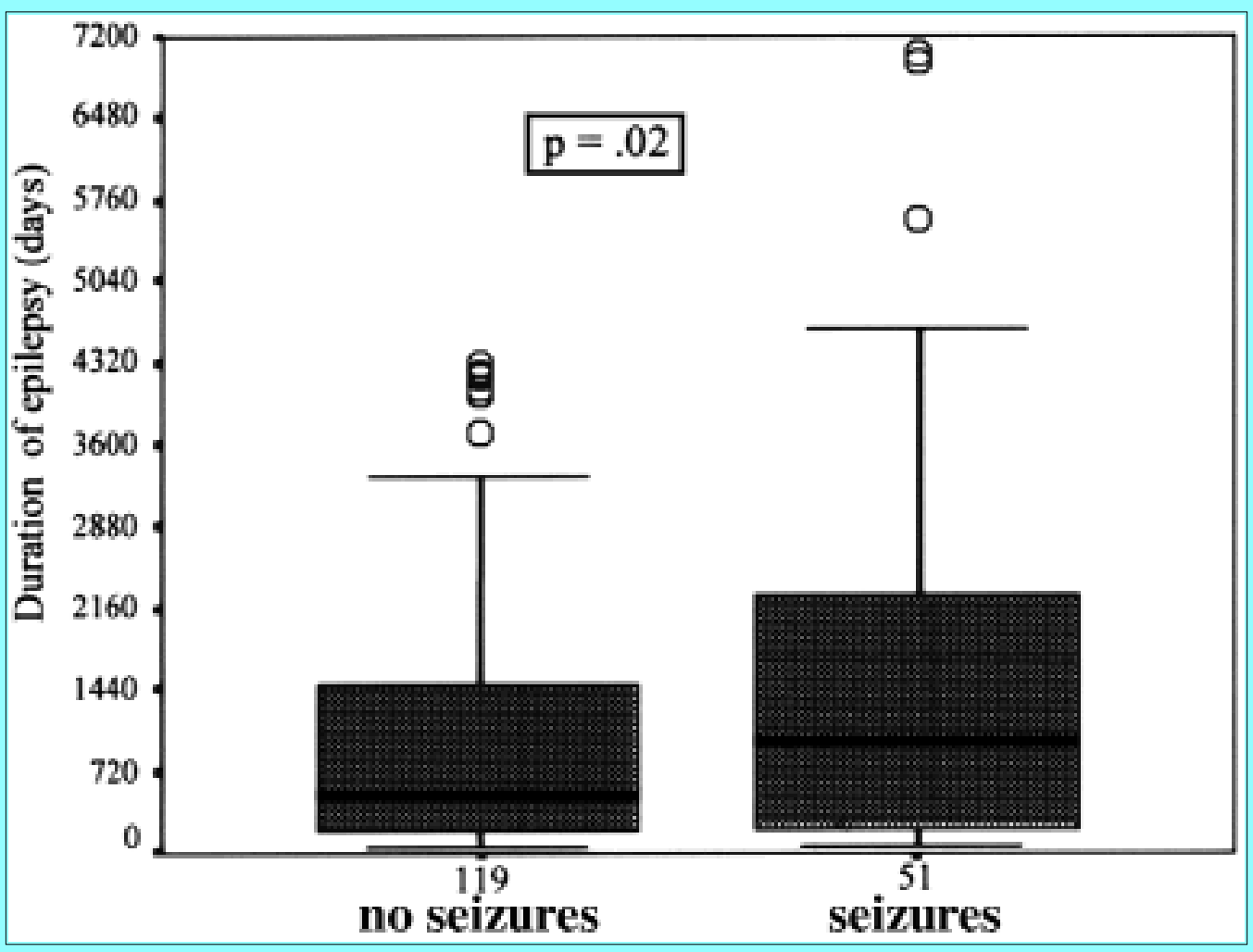

Fig. 6. Box-and-whiskers plot showing the correlation between the preoperative duration of epilepsy and the recurrence of seizures. The rectangle represents $50 \%$ of the cases, whereas the superior and inferior bars represent $25 \%$ each. The horizontal bold black bar represents the median.

Age at onset of seizures and loss of consciousness during seizures, although demonstrating a significant partial correlation with persistent seizures, were not included in the model. The other factors, namely, pathological characteristics, and location of the lesion were not found to enter into the model.

Cognitive Status. In this series effective surgical control of epilepsy was associated with an improvement in neurobehavioral status and rehabilitation. One-third of the children demonstrated developmental "catch up."

Better results were observed in children with psychological disorders, which were improved in $31 \%$ of the children overall. Children with hyperkinetic disorders benefited the most, with antisocial behavior such as rage attacks, impulsive behavior, and hyperactivity resolving postoperatively in $50 \%$ of the cases.

As for the beneficial effect of surgery on intellectual status, it was less frequently observed. Improvement in cognitive function was noted in $24.6 \%$ of the cases and was significantly inversely correlated with the preoperative duration of seizures; that is, the longer the preoperative duration of epilepsy, the less likelihood of there being an improvement after surgery. In five children deterioration occurred related to brain injury at the time of surgery.

Changes in schooling and academic performances reflect the changes in cognitive and psychological status. Improved school grades were noted in 10 children (7.6\%) of school age, with 95 children (61.3\%) having achieved normal schooling by the time of the study.

\section{DISCUSSION}

The potential adverse effect of epileptic activity on brain development is well recognized.[32] Because of 
the lack of consistently efficient anticonvulsive therapy and in spite of an incomplete understanding of the pathophysiology of epileptic activity related to focal lesions, surgical treatment has been attempted for a long time. Although most surgeons agree that the ultimate goal is removal of the epileptogenic area or interruption of connections between the epileptogenic focus and the rest of the brain, the necessary preoperative workup and the extent of surgical resection remain topics of debate. $[6,67,70,74]$

Before the introduction of computerized tomography and MR imaging there were no reliable neuroradiological investigations that could be performed to demonstrate focal lesions related to epilepsy.[7,14,16,51,57,71] This hindrance was coupled with a reluctance among clinicians to use "blind" invasive recordings on the developing brain of a noncooperative child. Currently, the previous pessimism accorded to the benefit of surgery is now being replaced by optimism because new technical advances have made surgery less invasive, more safe, and more predictable.[63] In the past decade we have seen considerable interest in the early surgical treatment of childhood epilepsy. It has been recognized that seizures starting in childhood, caused by known structural lesions and not controlled by medication, are unlikely to remit as the child matures.[79] In 1988 Aicardi[1] concluded: "It is this writer's opinion that surgical treatment of the epilepsies of childhood probably deserves more widespread use because of the poor response to drugs of many patients." For example, the rate of spontaneous remission rate in children undergoing medical therapy for the treatment of temporal lobe epilepsy has been reported to be lower than 20\%.[40,49,50] It has also been observed that, in a significant number of adult epileptic patients in whom subsequently surgery is performed, the initial seizures began in childhood. Furthermore, the risk of occurrence of psychiatric disorders in adult patients with childhood-onset epilepsy has been recently emphasized.[46] Similarly, the potential adverse effect of repeated seizures on the developing brain, the expectation of some developmental catch up, and the concept of young-brain equipotentiality (plasticity) that allows aggressive surger, have all been important issues in advocating early surgical treatment. $[19,20,23,33,73,80]$ In addition, the recognition of side effects caused by anticonvulsant therapy have positively influenced the trend for alternative management strategies.[24]

Debate, however, still persists on the indications and timing of surgery in children. It is doubtful whether selection criteria used to choose adult patients are suitable for children. Medically intractable epilepsy is the most discussed criterion. It can be assumed that, for a variety of lesions such as tumors or vascular malformations (half of the cases in this series), it is obvious that the risks induced by the progressive natural history outweigh the problem of epilepsy, thus mandating the need for surgery. Resection of the lesion, even when seizures are controlled by antiepileptic medication (14\% in this series), permits the accurate diagnosis of a process that carries its own inherent risks. $[28,65]$ In patients with nonproliferative lesions, which are well correlated with seizure characteristics and electrophysiological findings, the time that is lost while waiting to determine if the epilepsy is medical intractable may be tantamount to causing permanent neuropsychological impairment, as this report has clearly demonstrated. The longer the duration and the greater the seizure frequency, the higher the risk that the patient will incur neuropsychological impairment. It is difficult to see the virtue in postponing surgical treatment for several months in an infant who presents with daily seizures related to a focal lesion. Moreover, because of the loss of cerebral plasticity as the child matures, extensive surgery must be performed before 3 years of age; the sooner the better.

An IQ below 50 is generally considered to be a contraindication for surgery in epileptic adults. On the contrary, in epileptic children this should not be considered the case because a degree of developmental catch up and improvement in future quality of life can be expected, as occurred in one-third of the cases 
in this series. In addition, children with chronic epilepsy face many problems that prevent them from becoming independent and productive.[76] Aggressive outbursts, learning difficulties due to heavy medication, and difficulty being accepted by schoolmates and the community in general are among the obstacles.[78] Mizrahi and colleagues[56] noted that psychosocial, behavioral, and educational problems occurred more frequently in patients whose surgery was delayed until adulthood.

In childhood lesional epilepsy, there is still considerable debate over which type of preoperative workup is appropriate and preoperative strategies have not yet been standardized. The results of this series and other recent reports $[4,45,47,57]$ demonstrate that for children, good outcome can be expected when the decision for surgery is based on a good correlation among clinical, electrophysiological, and neuroradiological data. Further improvement in surgical selection of patients is likely to come after obtaining elaborate neuropsychological assessment, appropriate interictal and ictal video-EEG monitoring, and high-definition MR imaging examinations.[15,17,44] In patients who harbor radiologically well-defined lesions that correspond to clinical and electrophysiological findings, PET or SPECT may not be mandatory. However, these "functional" imaging tools could be used to obtain additional information that may influence the decision to perform epilepsy surgery when the clinical picture is less clear.[18-22,35,41,80] Invasive recordings, powerful but expensive and time-consuming techniques, are required when there is a disagreement among clinical, anatomical, and electrophysiological findings that is not resolved by ictal video-EEG and functional imaging (PET and SPECT).[62] Despite their invasive nature and inherent risk of causing morbidity, the indication for invasive recordings must be carefully evaluated for each individual case and should not be dismissed too easily. For instance, these types of recordings could be proposed not only for use in cases in which data obtained from clinical or other investigation modalities are in disagreement, but also for lesions adjacent to functional cortex or after failure of a lesionectomy. However, from a technical standpoint the use of these techniques (subdural recordings or stereoelectroencephalography) can be more difficult to achieve and to interpret after the patient has undergone a previous lesionectomy.

In treating epilepsy, the theoretical basis for the surgical resection of a lesion as a treatment modality is predicated on the correlation of the presenting features with the presence of epileptic foci that anatomically correspond to a gross structural lesion. Lesion surgery is based on the assumption that the seizure disorder and the presence of a lesion are interrelated. Several mechanisms have been theorized to explain the relationship between structural lesions and epilepsy: local neuronal injury, vascular compromise, interstitial edema, and neurotransmitter release. Specific morphological neuronal alterations have been observed in patients with brain tumors that are potentially epileptogenic.[5,13,34,57] It is of particular interest that in four of our cases, seizures recurred at the time of tumor relapse, which suggests a direct relationship between the lesion and the epileptogenic activity of the surrounding brain. Is it the lesion or the surrounding brain that is epileptogenic? This question has generated considerable debate in recent years. Several authors have considered that the outcome of surgery in patients with lesions was based on the nature of the underlying disease,[37] the completeness of the resection,[5] and the extent of the removal of the functionally defined, associated epileptic focus.[81] In this series, when taking into account in a multivariate analysis (logistic regression) all of the factors that potentially interact with outcome, subdivision into low-grade tumors, focal cortical dysplasia, DNETs, vascular abnormalities (cavernomas), or other disease types did not correlate with the outcome of surgery. However, among factors implicated in surgical management, the nature of the lesion is not meaningless. As in this series, excision of low-grade tumors without resection of surrounding cortex has been reported to be associated with good outcome; $[38,42,43,47,48]$ conversely, it is still debatable whether one should include in the 
surgical resection of cavernous angiomas the area of gliosis and hemosiderin deposition that usually surrounds the lesion.[36,57] In our series, any abnormal macroscopic tissue was removed despite lack of scientific evidence that this method improved results. Regardless of specific disease, the aim is always to remove as completely as possible the epileptogenic lesion without creating a new neurological deficit or worsening an existing one.

Based on analysis of our data, it is obvious that the quality and degree of surgery strongly influence the results. It is interesting to consider that $91 \%$ of patients with medically refractory epilepsy who had surgery with no additional surgical trauma were in Class Ia of Engel's system.

Nearly all patients who suffered a surgery-related injury still experienced seizures that were frequently worse when compared with preoperative status. Surgery-related injury had two potential causes; brain contusion by excessive retraction and/or vascular damage. Surgery-related injury was the main factor that correlated with a poor outcome.

As already stressed in the literature, complete resection of the lesion was associated with a higher chance of seizure-free outcome than incomplete resection.[34,64] Certainly our data suggest that complete resection, especially in children with large structural lesions, may increase the chance of a good outcome. The persistence of postsurgical seizures in 42 children in this series was highly correlated with incomplete resection of the structural abnormality as judged on $\mathrm{T}_{1}$ - and $\mathrm{T}_{2}$ - weighted MR imaging. Only $24 \%$ of the children in whom lesions were incompletely resected became seizure free. Incomplete resection can be due to poor lesion differentiation from the normal brain as observed in some cases of this series. It is probable that systems that provide "image-guided surgical capabilities" or intraoperative imaging will help to solve this problem. The second cause of incomplete resection in our series was extension of the structural abnormalities to a functional area; subpial transections have been reportedly successful in alleviating this problem.[27,29,53,60]

In this series, a prolonged preoperative seizure duration was found to correlate with a poor outcome in patients. Of concern have been the continuing influence of and pathological changes related to an active epileptogenic focus, " kindling," and the potentially time-related risk of secondary epileptogenesis. This presence of epileptogenic abnormalities that are remote from structural lesions has been well established. The postulate that the risk of secondary epileptogenesis correlates with a longer preoperative duration of epilepsy and a more frequent seizure occurrence calls for early evaluation of such children for surgical treatment.[58,59,69]

The location of the lesion, (temporal compared with extratemporal), has also been discussed in the literature as a factor that has potential influence on the outcome of epilepsy. We agree with the finding of Montes and colleagues,[57] that "temporal and extratemporal lesions do equally well after radical excision."

In this series, as in others, the postoperative EEG was a good predictor of patient outcome.[66] Patients whose EEG status normalized had a high probability of being seizure free $(94 \%$ of the children in this series). Conversely, those children in whom EEG abnormalities were demonstrated had a significant risk of seizure recurrence (one of two in this series).

The inclusion of a wide variety of lesions in this study could be called into question; however, such inclusiveness did not appear to exert any undue influence, either in terms of clinical presentation or outcome. A long-standing partial seizure disorder could be equally related to a low-grade brain tumor as 
well as to a focal cortical dysplasia or a dysembryoplastic lesion, all of which presented a similar clinical picture. It is also of interest to note that the results of statistical multivariate analysis did not show any correlation between the type of lesion and the outcome. This could be real or could be due to overweighting of other factors such as surgical damage, completeness of removal, and preoperative duration of seizures, which are sufficient to explain the results obtained in this series.

In the present study, one-fourth of the children who were seizure free or whose seizures were significantly reduced had improved developmental milestones and behavior. On the contrary, persistence of seizures led to further neuropsychological deterioration. Despite the relatively good results in seizure control, the percentage of patients who made postoperative cognitive and neuropsychological improvements ( $25 \%$ and $31 \%$, respectively) remained relatively low. Hence, is the goal of surgery to cause the patient to recover from already established deficits or to prevent further deterioration from occurring? Because of the limited cognitive and psychological improvement observed in patients after successful surgery, a multivariate analysis was conducted to identify those factors that may have correlated with the preoperative neuropsychological status. As shown previously, cognitive status was mainly influenced by the duration of seizures whereas psychological status depended on seizure frequency. The postoperative evolution was also found to be different. On the one hand, of those patients in whom a psychological deterioration occurred rapidly after onset of epilepsy, one-third improved postsurgery. On the other hand, cognitive disorders that appeared later in the course of the epilepsy (approximately 2 years) were less prone to improvement after successful surgery. It is probable that early surgery in the young child is the best guarantee of preventing neuropsychological deterioration.

\section{CONCLUSIONS}

Since the last decade, surgery has arisen as an alternative in the treatment of epilepsy in children with lesions. This is largely due to progress in neuroimaging and to a better understanding of the natural history of epilepsy in children. In addition, the risk of definitive neuropsychological deterioration in children who have experienced long-lasting seizures is now well recognized.

Surgical treatment is usually considered in patients suffering from medically intractable epilepsy that is associated with focal lesions. However, surgery can also be indicated in patients with less severe epilepsy even when medically controlled, when it is associated with lesions such as tumors or vascular malformations that have inherent risks of their own.

When there is a good correlation among seizure characteristics, neuroradiological findings, and location of the EEG abnormalities, it is probable, as demonstrated by this series and others, that favorable results can be obtained by performing resection of the lesion alone without invasive preoperative workup. This focal resection minimizes morbidity and the potential deleterious effects of more extensive brain-volume excisions and can encourage excellent rehabilitation of patients who are disabled by seizures and behavioral problems. The most important factor in determining successful seizure control after resection of lesion is a minimally traumatic operation and a complete removal of the lesion. Besides preventing chronic neuropsychological deterioration, shortening the duration of intractable epilepsy may reduce the incidence of the development of secondary epileptogenesis and improve the results of lesionectomy alone.

The indication for invasive monitoring could be limited to those cases with discordant data from clinical or other investigation modalities, as well as lesions adjacent to functional cortex. 


\section{References}

1. Aicardi J: Clinical approach to the management of intractable epilepsy. Dev Med Child Neurol 30:429-440, 1988

2. Aicardi J, Chevrie J: Atypical benign partial epilepsy of childhood. Dev Med Child Neurol 24:281-292, 1982

3. Al-Rodhan NR, Kelly PJ, Cascino DG, et al: Surgical outcome in computer-assisted stereotactic resection of intra-axial cerebral lesions for partial epilepsy. Stereotact Funct Neurosurg 58:172-177, 1992

4. Armon C, Radtke RA, Friedman AH, et al: Predictors of outcome of epilepsy surgery: multivariate analysis with validation. Epilepsia 37:814-821, 1996

5. Awad IA, Nayel MH: Epilepsy surgery: introduction and overview. Clin Neurosurg 38:493-513, 1992

6. Bancaud J, Talairach J, Munari C, et al: Introduction à l'étude clinique des crises épileptiques rétrorolandiques. Can J Neurol Sci 18 (Suppl 4):566-569, 1991

7. Barkovich AJ, Rowley HA, Andermann F: MR in partial epilepsy: value of high-resolution volumetric techniques. AJNR 16:339-343, 1995

8. Beardsworth ED, Zaidel DW: Memory for faces in epileptic children before and after brain surgery. J Clin Exp Neuropsychol 16:589-596, 1994

9. Beckung E, Uvebrant P, Hedstrom A, et al: The effects of epilepsy surgery on the sensorimotor function of children. Dev Med Child Neurol 36:893-901, 1994

10. Berger MS, Ghatan S, Geyer JR, et al: Seizure outcome in children with hemispheric tumors and associated intractable epilepsy: the role of tumor removal combined with seizure foci resection. Pediatr Neurosurg 17:185-191, 1991/92

11. Blume WT: Uncontrolled epilepsy in children. Epilepsy Res Suppl 5:19-24, 1992

12. Blume WT, Girvin JP, Kaufmann JC: Childhood brain tumors presenting as chronic uncontrolled focal seizure disorders. Ann Neurol 12:538-441, 1982

13. Cascino G: Intractable partial epilepsy: evaluation and treatment. Mayo Clin Proc 65:1578-1586, 1990

14. Cascino GD, Jack CR Jr, Hirschorn KA, et al: Identification of the epileptic focus: magnetic resonance imaging. Epilepsy Res Suppl 5:95-100, 1992

15. Cascino GD, Jack CR Jr, Parisi JE, et al: Magnetic resonance imaging-based volume studies in temporal lobe epilepsy: pathological correlations. Ann Neurol 30:31-36, 1991

16. Cascino GD, Jack CR Jr, Parisi JE, et al: MRI in the presurgical evaluation of patients with frontal lobe epilepsy and children with temporal lobe epilepsy: pathologic correlation and prognostic importance. Epilepsy Res 11:51-59, 1992 
17. Chuang SH, Otsubo H, Hwang P, et al: Pediatric magnetic source imaging. Neuroimaging Clin North Am 5:289-303, 1995

18. Chugani HT: PET in preoperative evaluation of intractable epilepsy. Pediatr Neurol 9:411-413, 1993

19. Chugani HT: The role of PET in childhood epilepsy. J Child Neurol 9 (Suppl 1):S82-S88, 1994 20. Chugani HT, Shields WD, Shewmon DA, et al: Infantile spasms: I. PET identifies focal cortical dysgenesis in cryptogenic cases for surgical treatment. Ann Neurol 27:406-413, 1990

21. Cross JH, Gordon I, Jackson GD, et al: Children with intractable focal epilepsy: ictal and interictal 99TcM HMPAO single photon emission computed tomography. Dev Med Child Neurol 37:673-681, 1995

22. Cummings TJ, Chugani DC, Chugani HT: Positron emission tomography in pediatric epilepsy. Neurosurg Clin North Am 6:465-472, 1995

23. Dam M: Children with epilepsy: the effect of seizures, syndromes, and etiological factors on cognitive functioning. Epilepsia 31 (Suppl 4):S26-S29, 1990

24. Dam M: Side-effects of drug treatment in epilepsy. Acta Neurol Scand Suppl 117:34-41, 1988

25. Daumas-Duport C, Scheithauer BW, Chodkiewicz JP, et al: Dysembryoplastic neuroepithelial tumor: a surgically curable tumor of young patients with intractable partial seizures. Report of thirty-nine cases. Neurosurgery 23:545-556, 1988

26. Devinsky O, Pacia S: Epilepsy surgery. Neurol Clin 11:951-971, 1993

27. Devinsky O, Perrine K, Vazquez B, et al: Multiple subpial transactions in the language cortex. Brain 117:255-265, 1994

28. Dirks PB, Jay V, Becker LE, et al: Development of anaplastic changes in low-grade astrocytomas of childhood. Neurosurgery 34:68-78, 1994

29. Dogali M, Devinsky O, Luciano D, et al: Invasive intracranial monitoring, cortical resection and multiple subpial transection for the control of intractable complex partial seizure of cortical onset. Stereotact Funct Neurosurg 62:222-225, 1994

30. Drake JM, Prudencio J, Holowaka S, et al: Frameless stereotaxy in children. Pediatr Neurosurg 20:152-159, 1994

31. Duchowny M: Epilepsy surgery in children. Curr Opin Neurol 8:112-116, 1995

32. Farwell JR, Dodrill CB, Batzel LW: Neuropsychological abilities of children with epilepsy. Epilepsia 26:395-400, 1985

33. Finger S, Wolf C: The 'Kennard effect' before Kennard. The early history of age and brain lesions. Arch Neurol 45:1136-1142, 1988

34. Fish DR, Smith SJ, Quesney LF, et al: Surgical treatment of children with medically intractable frontal or temporal lobe epilepsy: results and highlights of 40 years' experience. Epilepsia 34:244-247, 
35. Gaillard WD, White S, Malow B, et al: FDG-PET in children and adolescents with partial seizures: role in epilepsy surgery evaluation. Epilepsy Res 20:77-84, 1995

36. Giulioni M, Acciarri N, Padovani R, et al: Results of surgery in children with cerebral cavernous angiomas causing epilepsy. Br J Neurosurg 9:135-141, 1995

37. Goldring S: Pediatric epilepsy surgery. Epilepsia 28 (Suppl 1):82-102, 1987

38. Goldring S, Rich KM, Picker S: Experience with gliomas in patients presenting with a chronic seizure disorder. Clin Neurosurg 33:15-42, 1986

39. Green RC, Adler JR, Erba G: Epilepsy surgery in children. J Child Neurol 3:155-166, 1988

40. Harbord MG, Manson JI: Temporal lobe epilepsy in childhood: reappraisal of etiology and outcome. Pediatr Neurol 3:263-268, 1987

41. Harvey AS, Berkovic SF: Functional neuroimaging with SPECT in children with partial epilepsy. J Child Neurol 9 (Suppl 1):S71-S81, 1994

42. Hirsch JF: Epilepsy and brain tumours in children. J Neuroradiol 16:292-300, 1989

43. Hirsch JF, Sainte Rose C, Pierre-Kahn A, et al: Benign astrocytic and oligodendrocytic tumors of the cerebral hemispheres in children. J Neurosurg 70:568-572, 1989

44. Holmes GL: Surgery for intractable seizures in infancy and early childhood. Neurology 43 (11 Suppl 5):S28-S37, 1993

45. Holmes MD, Dodrill CB, Ojemann LM, et al: Five-year outcome after epilepsy surgery in nonmonitored and monitored surgical candidates. Epilepsia 37:748-752, 1996

46. Jalava M, Sillanpaa M: Concurrent illnesses in adults with childhood-onset epilepsy: a population-based 35-year follow-up study. Epilepsia 37:1155-1163, 1996

47. Kim S, Wang K, Cho B: Intractable seizures associated with brain tumor in childhood: lesionectomy and seizure outcome. Childs Nerv Syst 11:634-638, 1995

48. Kirkpatrick PJ, Honavar M, Janota L, et al: Control of temporal lobe epilepsy following en bloc resection of low-grade tumors. J Neurosurg 78:19-25, 1993

49. Kotagal P, Luders HO: Recent advances in childhood epilepsy. Brain Dev 16:1-15, 1994

50. Kotagal P, Rothner AD, Erenberg G, et al: Complex partial seizures of childhood onset. A five-year follow-up study. Arch Neurol 44:117711-1180, 1987

51. Kuzniecky R: MRI in cerebral developmental malformations and epilepsy. Magn Reson Imaging 13:1137-1145, 1995

52. Leung SY, Gwi E, Ng HK, et al: Dysembryoplastic neuroepithelial tumor. A tumor with small neuronal cells resembling oligodendroglioma. Am J Surg Pathol 18:604-614, 1994

53. Liu Z, Zhao Q, Li S, et al: Multiple subpial transection for treatment of intractable epilepsy. Chinese 
Med J 108:539-541, 1995

54. Madsen JR, Adelson PD, Haglund MM: The future of pediatric epilepsy surgery. Signposts and science. Neurosurg Clin North Am 6:589-597, 1995

55. Meyer FB, Marsh WR, Laws ER Jr, et al: Temporal lobectomy in children with epilepsy. J Neurosurg 64:371-376, 1986

56. Mizrahi EM, Kellaway P, Grossman RG, et al: Anterior temporal lobectomy and medically refractory temporal lobe epilepsy of childhood. Epilepsia 31:302-312, 1990

57. Montes JL, Rosenblatt B, Farmer JP, et al: Lesionectomy of MRI detected lesions in children with epilepsy. Pediatr Neurosurg 22:167-173, 1995

58. Morrell F: Secondary epileptogenesis in man. Arch Neurol 42:318-35, 1985

59. Morrell F: Varieties of human secondary epileptogenesis. J Clin Neurophysiol 6:227-275, 1989

60. Morrell F, Whisler WW, Bleck TP: Multiple subpial transection: a new approach to the surgical treatment of focal epilepsy. J Neurosurg 70:231-239, 1989

61. Morrison G, Duchowny M, Resnick T, et al: Epilepsy surgery in childhood. A report of 79 patients. Pediatr Neurosurg 18:291-297, 1992

62. Ojemann GA: Different approaches to resective epilepsy surgery: standard and tailored. Epilepsy Res Suppl 5:169-174, 1992

63. Olivier A: Risk and benefit in the surgery of epilepsy: complications and positive results on seizures tendency and intellectual function. Acta Neurol Scand Suppl 117:114-121, 1988

64. Palmini A, Andermann F, Olivier A, et al: Focal neuronal migration disorders and intractable partial epilepsy: results of surgical treatment. Ann Neurol 30:750-757, 1991

65. Pascual-Castroviejo I, Garcia Blasquez M, Gutierrez Molina M, et al: 24-year preoperative evolution of a temporal astrocytoma. Childs Nerv Syst 12:417-420, 1996

66. Patrick S, Berg A, Spencer S: EEG and seizure outcome after epilepsy surgery. Epilepsia 36:236-240, 1995

67. Rasmussen T: Cortical resection in the treatment of focal epilepsy. Adv Neurol 8:139-154, 1975

68. Rasmussen T: Surgery of epilepsy associated with brain tumors. Adv Neurol 8:227-239, 1975

69. Rougier A: The epileptic focus versus the pathological focus. Acta Neurochir Suppl 50:1-5, 1990

70. Spencer SS: Depth electroencephalography in selection of refractory epilepsy for surgery. Ann Neurol 9:207-214, 1981

71. Spencer SS: MRI and epilepsy surgery. Neurology 45:1248-1250, 1995

72. Storrs BB: Surgery for epilepsy in children. J South Carolina Med Assoc 88:256-257, 1992

73. Sur M, Garraghty PE, Roe AW: Experimentally induced visual projections into auditory thalamus 
and cortex. Science 242:1437-1441, 1988

74. Talairach J, Bancaud J, Bonis A, et al: Functional stereotaxic exploration of epilepsy. Confin Neurol 22:328-331, 1962

75. Taratuto AL, Pomata H, Sevlever G, et al: Dysembryoplastic neuroepithelial tumor: morphological, immunocytochemical, and deoxyribonucleic acid analyses in a pediatric series. Neurosurgery 36:474-481, 1995

76. Taylor DC, Lochery M: Behavioral consequences of epilepsy in children. Developing a psychosocial vocabulary. Adv Neurol 55:153-162, 1991

77. Vickrey BG, Hays RD, Engel J Jr, et al: Outcome assessment for epilepsy surgery: the impact of measuring health-related quality of life. Ann Neurol 37:158-166, 1995

78. Wyllie E: Cortical resection for children with epilepsy. Perspectives in pediatrics. Am J Dis Child 145:314-320, 1991

79. Wyllie E: Surgery for catastrophic localization-related epilepsy in infants. Epilepsia 37 (Suppl 1):S22-S25, 1996

80. Wyllie E, Comair YG, Kotagal P, et al: Epilepsy surgery in infants. Epilepsia 37:625-637, 1996

81. Wyllie E, Luders H, Morris HH III, et al: Clinical outcome after complete or partial cortical resection for intractable epilepsy. Neurology 37:1634-1641, 1987

82. Wyllie E, Rothner AD, Luders H: Partial seizures in children: clinical features, medical treatment, and surgical considerations. Pediatr Clin North Am 36:43-64, 1989

Manuscript received December 15, 1998.

Accepted in final form January 20, 1999.

Address reprint requests to: Christian Sainte-Rose, M.D., Service de Neurochirurgie Pédiatrique, Hôpital Necker-Enfants Malades, 149, rue de Sèvres, 75015 Paris, France. email:

christian.sainte-rose@nck.ap-hop-paris.fr. 